\title{
Influência das Estações do Ano nas Características Seminais de Carneiros
}

\section{Leira Tutida ${ }^{2}$, Orlando Rus Barbosa ${ }^{3}$, Elias Nunes Martins ${ }^{3}$, Francisco de Assis Fonseca de Macedo $^{3}$, Márcio José da Rocha Roman ${ }^{4}$, Sandra M. Simonelli ${ }^{4}$}

\begin{abstract}
RESUMO - O objetivo deste estudo foi avaliar o efeito das estações do ano sobre motilidade progressiva (MP), concentração espermática, vigor e volume de sêmen de carneiros das raças Bergamácia, Corriedale e Hampshire Down, com 3 a 4 anos de idade, no período de março de 1996 a março de 1997. Foram utilizados vinte e quatro carneiros, sendo nove da raça Bergamácia (Be), oito Corriedale (Co) e sete Hampshire Down (Hd). A coleta do sêmen e o registro das variáveis climáticas: velocidade do vento ( $v$ ), temperatura do ar (Ta), temperatura do globo (Tg) e umidade relativa do ar (Ur) foram feitos mensalmente. Para MP, as raças Be e Co apresentaram maiores valores no verão, com decréscimo no outono e inverno e novo aumento na primavera. A raça Hd apresentou maiores valores no verão, decrescendo nas outras estações. Para vigor espermático, as raças Be e Co apresentaram maiores valores no verão, decrescendo no outono e inverno, e novo aumento na primavera, mas não teve efeito para Hd. Para volume de sêmen, as raças Co e Hd apresentaram maiores valores no verão, com queda no outono e inverno e novo aumento na primavera. Não houve efeito das estacões sobre o vigor espermático para raça Be. A concentração espermática não foi influenciada pela estação, permanecendo constante para as três raças estudadas. A $v$ foi a que apresentou maior influência sobre as características de sêmen, seguida da Ta e Tg e Ur. As raças Be e Hd apresentaram melhores respostas que a Co, para as características de sêmen, resistindo melhor ao ambiente.
\end{abstract}

Palavras-chave: carneiro, estação do ano, sêmen

\section{Influence of the Season of Year on the Seminal Characteristics of Rams}

\begin{abstract}
The objective of this study was to evaluate the effect of the season of the year on the sperm progressive motility (PM), vigor, volume of semen and sperm concentration of rams from the Bergamacia, Corriedale and Hampshire Down (Hd) breeds, with three-four years of age, from March/96 to March/97. Twenty-four rams nine Bergamacia (Be), eight Corriedale (Co) and seven Hampshire Down (Hd) were used. The semen collection and the climatic variables: wind speed (ws), air temperature (Ta), globe temperature (GT) and air relative humidity (RH) were taken monthly. For PM, the Be and Co breeds showed higher values during the summer, decreasing in the autumn and in the winter and increase again in the spring. The Hd breed presented higher values during the summer, decreasing in other seasons. For spermatic vigor, Be and Co breeds showed higher values during the summer, decreased in the autumn and in the winter, and increased again in spring. There was no effect of season on the spermatic vigor of Hd breed. For semen volume, Co and Hd showed higher values during summer, falling in autumn and in winter, and rising in spring. The spermatic concentration was not affected by the season, maintaining constant for the three studied breeds. The ws was the climatic variable that showed the highest influence on semen characteristics, followed by Ta, GT and RH. The Be and Hd breeds showed better results than Co breed for the semen characteristics, resisting better to the environment.
\end{abstract}

Key Words: ram, season, semen

\section{Introdução}

A quantidade e a qualidade do sêmen de ovinos variam com as estações do ano e o grau dessa dependência estacional varia de acordo com a espécie, a raça e a localidade. Nas zonas temperadas há grande variação no sêmen produzido por ovinos das raças européias e americanas. Durante o verão, as características do sêmen são inferiores e, em muitos casos, não há ejaculação.
Os cruzamentos, por intermédio da inseminação artificial com sêmen importado, têm sido aceitos como forma de aumentar a produtividade dos animais nativos. Entretanto, os animais exóticos introduzidos em áreas de clima diferente ao de origem exibem deterioração na sua performance reprodutiva, observada pelas alterações na qualidade e nos constituintes bioquímicos do sêmen (RAO et al.,1983).

Nos ovinos, a qualidade do sêmen e a fertilidade do macho tendem a diminuir durante os meses mais

\footnotetext{
1 Parte da dissertação de Mestrado em Zootecnia, apresentada à Universidade Estadual de Maringá - UEM, pelo primeiro autor.

${ }^{2}$ Estudante do Curso de Mestrado em Zootecnia da UEM - Maringá, PR.

3 Professor do Departamento de Zootecnia da UEM - Maringá, PR.

${ }^{4}$ Estudante de graduação - UEM, PR - Bolsista do CNPq.
} 
quentes (verão), possivelmente devido aos efeitos estacionais no eixo hipotálamo-hipófise ou ao efeito direto da temperatura nos testículos e epidídimo (PINEDA, 1989).

O ambiente fótico influencia a função reprodutiva no macho, alterando a produção de hormônios gonadotróficos pela hipófise e, conseqüentemente, o desenvolvimento gonadal. Desse modo, o efeito da estação pode ser atribuído à temperatura do ar, à disponibilidade e à qualidade da forragem, juntamente com os regimes curtos de luz (CURTIS,1983; CHEMINEAU, 1994).

Assim, o presente trabalho teve por objetivo verificar o efeito das estações do ano na qualidade do sêmen de carneiros das raças Bergamácia, Corriedale e Hampshire Down, bem como verificar o comportamento adaptativo destas raças nas condições ambientais em que são criadas.

\section{Material e Métodos}

O experimento foi conduzido no setor de ovinocultura da Fazenda Experimental de Iguatemi da Universidade Estadual de Maringá (UEM) - PR, no período de março de 1996 a março de 1997.

Foram utilizados 24 carneiros, de 3 a 4 anos de idade, sendo sete Hampshire Down (Hd), oito Corriedale (Co) e nove Bergamácia $(\mathrm{Be})$, pertencentes ao rebanho da UEM/Campus Arenito Cidade Gaúcha, selecionados por meio de exames andrológicos, conforme MIES FILHO (1987). Estes animais foram mantidos, durante o período experimental, em regime de semi confinamento, sob luminosidade natural, alimentados a pasto, com suplementação de $0,5 \mathrm{~kg} / \mathrm{cabeça} /$ dia de concentrado com $14 \%$ de proteína bruta, mais mistura mineral e água ad libitum.

O controle de ecto e endoparasitas foi realizado por meio das contagens mensais de ovos nas fezes e aplicação de vermífugo nos animais que apresentavam contagem superior a 500 ovos por grama de fezes.

Foram realizadas duas tosquias, uma no início (jan/96) e outra durante (jan/97) o experimento.

As coletas de sêmen foram efetuadas a cada 30 dias, durante 12 meses, por meio de vagina artificial, modelo curto, usando-se como manequim uma ovelha estrogenizada, conforme recomendação de MIES FILHO (1987). O sêmen coletado foi mantido em banho-maria a $37^{\circ} \mathrm{C}$, efetuando-se a análise macroscópica de volume e análises microscópicas de motilidade progressiva (MP), segundo MOULE (1965), vigor e concentração espermática. Para concentração $\left(\mathrm{mm}^{3}\right)$, foram utilizadas câmaras de Neubauer dupla, "improved Germany”, para contagem de células sangüíneas, após diluição em solução de cloreto de sódio a 3\%, na proporção de 1:400.

Nos dias reservados a essas determinações, a cada hora foram obtidas a temperatura (Ta) e umidade do ar (Ur), por intermédio de um psicrômetro nãoventilado de bulbo seco e bulboúmido; a velocidade do vento $(v)$, por intermédio de um catatermômetro, conforme SILVA e BRASIL (1986); e a temperatura do globo negro ao sol ( $\mathrm{Tg}$ ), por intermédio de um globo de Vernon de 0,15 m de diâmetro (SILVA, 1989), para medir a carga térmica radiante naquele momento.

Médias meteorológicas mensais referentes aos 12 meses do experimento foram obtidas no campus da UEM, em Maringá, para correlações com as medidas fisiológicas. Procurou-se, na análise, determinar a variação anual das diversas variáveis, frente à sazonalidade climática.

A análise dos dados consistiu de análise de regressão e teste de identidade de modelos, segundo GRAYBILL (1976), seleção de variáveis por intermédio do "stepwise", conforme DRAPER e SMITH (1981), e análise de trilha, segundo SILVA (1980) e CRUZ e REGAZZI (1994).

Para a análise de regressão e teste de identidade de modelos, foi utilizado o seguinte modelo matemático:

$\mathrm{Y}_{\mathrm{ijk}}=\mu+\mathrm{R}_{\mathrm{i}}+\mathrm{b}_{1 \mathrm{i}}\left(\mathrm{M}_{\mathrm{k}}-\mathrm{M}\right)+\mathrm{b}_{2 \mathrm{i}}\left(\mathrm{M}_{\mathrm{k}}-\mathrm{M}\right)^{2}$ $+\mathrm{b}_{3 \mathrm{i}}\left(\mathrm{M}_{\mathrm{k}}-\mathrm{M}\right)^{3}+\mathrm{e}_{\mathrm{ijk}}$

em que

$\mathrm{Y}_{\mathrm{ijk}}=$ observação da característica seminal $\mathrm{k}$ da raça $\mathrm{i}$, no mês $\mathrm{j}$;

$\mu=$ constante geral;

$\mathrm{R}_{\mathrm{i}}=$ efeito da raça $\mathrm{i}, \mathrm{i}=1 ; 2 ; 3$;

$\mathrm{b}_{1 \mathrm{i}}=$ coeficiente de regressão linear da característica seminal da raça $\mathrm{i}$, em função do mês $\mathrm{j}$;

$\mathrm{b}_{2 \mathrm{i}}=$ coeficiente de regressão quadrática da característica seminal da raça $i$, em função do mês j;

$\mathrm{b}_{3 \mathrm{i}}=$ coeficiente de regressão cúbica da característica seminal da raça i, em função do mês j;

$\mathrm{M}_{\mathrm{k}}=$ valor de identificação do mês $\mathrm{k}, \mathrm{k}=1 ; 2 ; 3 ; . . ; 12$;

$\mathrm{M}=$ valor médio da variável mês; $\mathrm{e}$

$\mathrm{e}_{\mathrm{ijk}}=$ erro aleatório associado a cada observação $Y_{\mathrm{ijk}}$.

Quando encontrado efeito significativo para as raças, foi aplicado o teste Tukey, a 5\% de probabilidade, visando identificar as diferenças entre as médias das raças. 
Rev. bras. zootec.

\section{Resultados e Discussão}

Pela análise da Tabela 1, observa-se que a raça apresentou efeito $(\mathrm{P}<0,01)$ para MP e o mês teve efeito quadrático $(\mathrm{P}<0,01)$ para as raças $\mathrm{Be}$ e Co e efeito linear $(\mathrm{P}<0,05)$, para a raça $\mathrm{Hd}$. $\mathrm{O}$ vigor espermático foi afetado $(\mathrm{P}<0,01)$ pela raça e o mês teve efeito quadrático $(\mathrm{P}<0,01)$ para as raças Be e Co. Para o volume, houve efeito da raça e o mês teve efeito quadrático $(\mathrm{P}<0,01)$ para as raças Co e Hd. Não houve efeito de raça e mês para a característica concentra- ção do sêmen.

Na Tabela 2, são apresentadas as médias estimadas das características do sêmen das três raças de carneiros estudadas.

Observa-se que as raças Be e Hd apresentaram características de MP, vigor e volume semelhantes, porém diferiram $(\mathrm{P}<0,05)$ do apresentado pela raça Co. Segundo DERIVAUX (1976), um sêmen de boa qualidade deve apresentar MP de 60 a $70 \%$ e vigor de 4 a 5 pontos. As raças Be e Hd apresentaram MP dentro dos valores citados, porém com vigor inferior,

Tabela 1 - Resumo da análise de variância dos dados de motilidade progressiva (MP), vigor, volume e concentração do sêmen de carneiros das raças Bergamácia, Corriedale e Hampshire Down, em função do mês

Table 1 - Summary of the analysis variance of progressive motility (PM), vigor, volume and sperm concentration of rams of Bergamacia, Corriedale and Hampshire Down breeds in function of month

\begin{tabular}{|c|c|c|c|c|c|}
\hline \multirow{3}{*}{$\begin{array}{l}\text { Fonte de variação } \\
\text { Source of variation }\end{array}$} & \multirow{3}{*}{$\begin{array}{r}\mathrm{gl}^{1} \\
d f\end{array}$} & \multicolumn{3}{|c|}{$\begin{array}{l}\text { Quadrado médio } \\
\text { Mean square }\end{array}$} & \multirow{3}{*}{$\begin{array}{l}\text { Concentração } \\
\text { Concentration }\end{array}$} \\
\hline & & MP & Vigor & Volume & \\
\hline & & $P M$ & & & \\
\hline Raça & 2 & $9459,249 * *$ & $23,51073 * *$ & $6,731287 * *$ & \\
\hline $\begin{array}{l}\text { Breed } \\
\text { Mês Be/Co - Linear }\end{array}$ & 1 & & & - & \\
\hline $\begin{array}{l}\text { Month Be/Co - Linear } \\
\text { Mês Be/Co - Quad. } \\
\text { Month Be/Co - Quadratic }\end{array}$ & 1 & $17503,89 * *$ & $44,19013 * *$ & - & \\
\hline $\begin{array}{l}\text { Mês Hd - Linear } \\
\text { Month Hd - Linear }\end{array}$ & 1 & $5132,655^{*}$ & - & - & \\
\hline $\begin{array}{l}\text { Mês Co/Hd - Linear } \\
\text { Month Co/Hd - Linear }\end{array}$ & 1 & - & - & $\mathrm{ns}$ & \\
\hline $\begin{array}{l}\text { Mês Co/Hd - Quad. } \\
\text { Month Co/Hd - Quadratic }\end{array}$ & 1 & - & - & $6,281604 * *$ & \\
\hline $\begin{array}{l}\text { Mês Be/Co/Hd - Linear } \\
\text { Month Be/Co/Hd - Linear }\end{array}$ & - & - & - & - & \\
\hline $\begin{array}{l}\text { Mês Be/Co/Hd - Quad. } \\
\text { Month Be/Co/Hd - Quadratic }\end{array}$ & - & - & - & - & \\
\hline $\begin{array}{l}\text { Mês Be/Co/Hd - Cúbico } \\
\text { Month Be/Co/Hd - Cubic }\end{array}$ & - & - & - & - & \\
\hline $\begin{array}{l}\text { Resíduo } \\
\text { Error }\end{array}$ & - & 983,9445 & 2,394660 & 0,3628720 & 4,653965 \\
\hline $\begin{array}{l}\text { gl Resíduo } \\
\text { df Error }\end{array}$ & - & 249 & 250 & 250 & 249 \\
\hline $\mathrm{CV}(\%)$ & & 46,796 & 47,889 & 53,993 & 75,511 \\
\hline
\end{tabular}

** $(P<0,01) *(P<0,05)$

Tabela 2 - Médias estimadas dos dados de motilidade progressiva (MP), vigor e volume do sêmen de carneiros das raças Bergamácia, Corriedale e Hampshire Down

Table 2 - Estimated means of sperm progressive motility (PM), vigor and volume of rams of Bergamacia, Corriedale and Hampshire Down breeds

\begin{tabular}{lccc}
\hline Raça & $M P(\%)$ & Vigor ( 0 a 5 pontos $)$ & Volume $(\mathrm{mL})$ \\
Breed & $P M$ & Vigor (score - 0 a 5) & \\
\hline Bergamácia & $66,50^{\mathrm{a}}$ & $3,26^{\mathrm{a}}$ & $1,21^{\mathrm{a}}$ \\
Corriedale & $46,96^{\mathrm{b}}$ & $2,25^{\mathrm{b}}$ & $0,62^{\mathrm{b}}$ \\
Hampshire Down & $64,12^{\mathrm{a}}$ & $2,97^{\mathrm{a}}$ & $1,07^{\mathrm{a}}$ \\
\hline
\end{tabular}

Médias, na coluna, seguidas de letras diferentes são diferentes $(P>0,05)$ pelo teste Tukey.

Means, within a column, followed by different letters are different $(P<.05)$ by Tukey test. 
enquanto a raça Co apresentou MP e vigor inferiores aos citados na literatura.

O volume observado nas raças estudadas está dentro do obtido por HULET e SHELTON (1982), que variou de 0,3 a 2,0 mL.

Os valores observados apresentaram-se, às vezes, abaixo dos citados na literatura, devido à individualidade dos animais, pois alguns deles apresentaram falha na ejaculação.

Segundo FOOTE (1982), a estação, a idade, o tamanho, a raça e a freqüência de coleta influenciam as características seminais. O volume de sêmen pode variar entre espécie, dentro da mesma espécie, segundo o estado fisiológico do macho, o número de saltos e o método de coleta, higiene e alimentação (DERIVAUX, 1976).

Na Figura 1, observa-se que, no comportamento da MP, em função do mês, as raças Be e Co apresentaram maiores valores durante o verão, com queda no outono e inverno e aumento gradual na primavera. A raça $\mathrm{Hd}$ apresentou maiores valores durante o verão, com queda nas demais estações.

Estes resultados estão de acordo com os encontrados por MICKELSEN et al. (1981), que também obtiveram maiores valores na primavera (79 e 75\%) e verão (77 e $87 \%$ ) e menores valores no outono (68 e 74\%) e inverno (61 e 67\%), em carneiros Suffolk e Lincoln, respectivamente.Contudo, diferem dos obtidos por DUTT e BUSH (1955) e DUTT e SIMPSON (1957), nos quais a MP foi reduzida em carneiros Southdown (66 para 33\%), quando mantidos em temperaturas de $31,5^{\circ} \mathrm{C}$, no verão.

Alguns autores verificaram maior MP (67\%) no sêmen de carneiros submetidos a temperaturas baixas (BROOKS e ROSS, 1962), mas outros, como DUFOUR et al. (1984), observaram alta MP no outono (50\%); entretanto, MITTAL e GHOSH (1979) não encontraram diferença nesta variável.

Assim, em virtude de a característica do sêmen variar de acordo com a estação do ano e o grau de tal dependência estacional ocorrer de acordo com a espécie, a raça e a localidade, é de se esperar tais variações, haja visto que a individualidade dos animais foi bastante marcante durante o período de coleta e, em muitos casos, alguns animais não apresentaram ejaculação.

Quando se analisa o comportamento do volume de sêmen em função do mês (Figura 2), observa-se que as raças Co e Hd apresentaram maiores valores no verão, seguido de queda no outono e no inverno, com aumento na primavera. Estes resultados estão

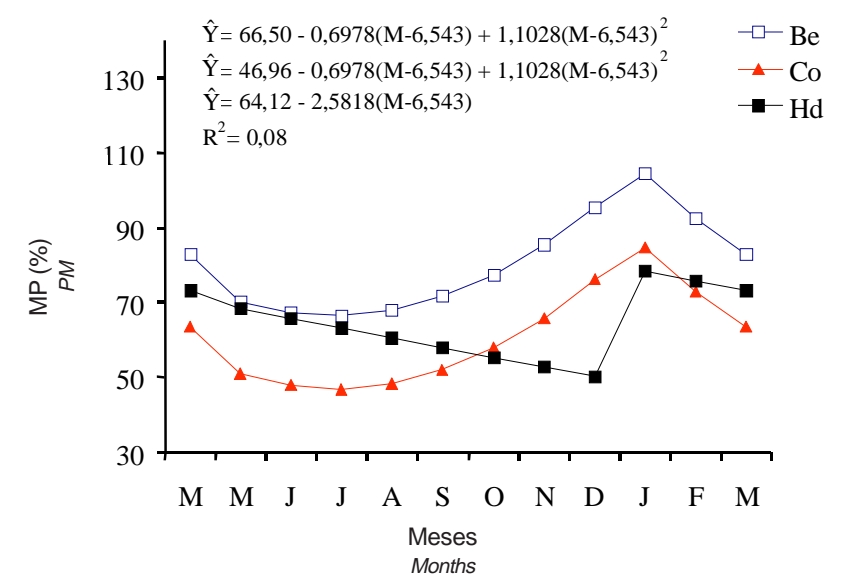

Figura 1 - Comportamento da MP do sêmen de carneiros das raças $\mathrm{Be}$, Co e $\mathrm{Hd}$ em relação aos meses do ano.

Figure 1 - Behaviour of sperm PM of rams of $\mathrm{Be}$, Co and $\mathrm{Hd}$ breeds, on months of the year.

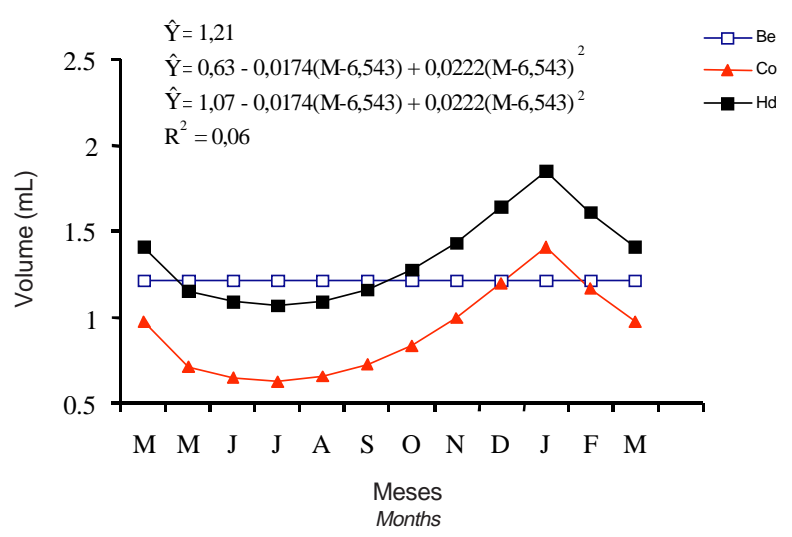

Figura 2 - Comportamento do volume do sêmen de carneiros das raças $\mathrm{Co}, \mathrm{Hd}$ e $\mathrm{Be}$, em relação aos meses do ano.

Figure 2 - Behaviour of semen volume of rams of $\mathrm{Co}, \mathrm{Hd}$ and $\mathrm{Be}$ breeds on months of the year.

de acordo com os encontrados por BOLAND et al. (1985) e BROOKS e ROSS (1962), que também obtiveram maiores valores no verão em carneiros Suffolk, Texel e Dorset Horn $(1,5 \pm 0,06 \mathrm{~mL})$ e Hampshire $(1,29 \mathrm{~mL})$, respectivamente.

CHAHAL et al. (1979) também observaram maior volume de sêmen em carneiros Corriedale no verão $(1,17 \mathrm{~mL})$ e estação chuvosa $(0,99 \mathrm{~mL})$ que no inverno $(0,58 \mathrm{~mL})$ e na primavera $(0,78 \mathrm{~mL})$, atribuindo-os à possível atividade endócrina dependente da temperatura dos testículos, que pode ser responsável pelo aumento na secreção das glândulas acessórias. Por outro lado, MITTAL e GHOSH (1979) não encontraram alteração estacional no volume do sêmen de carneiros Corriedale mantidos sob ambiente 
Rev. bras. zootec.

natural $\left(31,5^{\circ} \mathrm{C}\right)$ durante três semanas.

$\mathrm{Na}$ Figura 3 observa-se o comportamento do vigor do sêmen em função do mês. As raças Be e Co apresentaram maiores valores de vigor no verão, com queda no outono e inverno e aumento na primavera.

Segundo DERIVAUX (1976), um sêmen de boa qualidade deve apresentar vigor de 4 a 5 pontos. Desse modo, as raças $\mathrm{Be}$ e $\mathrm{Co}$, no verão e na primavera, tiveram valores próximos ao padrão estabelecido, que, por sua vez, coincide com a entrada dos animais na reprodução.

Com objetivo de melhor detalhar a ação das variáveis climáticas ( Ta, Tg, v e Ur) sobre as características seminais (MP, vigor e volume), efetuou-se o método da análise de trilha.

A Figura 4 mostra os efeitos das variáveis climáticas sobre o volume do sêmen. A Ta apresentou efeito direto de 0,78 , enquanto seus efeitos indiretos via $\mathrm{Tg}, v$ e Ur foram de $-0,391 ;-0,137$; e 0,026 , respectivamente. A $v$ apresentou efeito direto de 0,763 , enquanto seus efeitos indiretos via $\mathrm{Ta}, \mathrm{Tg}$ e $\mathrm{Ur}$ foram de 0,$14 ;-0,13$; e 0,002 , respectivamente. A Tg apresentou efeito direto de $-0,665$, enquanto seus efeitos indiretos via Ta, $v$ e Ur foram de 0,$459 ;-0,149$; e 0,128 , respectivamente. A Ur apresentou efeito direto de $-0,355$, enquanto seus efeitos indiretos via $\mathrm{Tg}$, Ta e $v$ foram de 0,$24 ;-0,057$; e 0,005 , respectivamente. A $v$ apresentou alto efeito direto e correlação com o volume, determinando a variação. Tanto a Ta como a $\mathrm{Tg}$ apresentaram alto efeito direto e baixa correlação, sendo mais benéficas para as estimativas quando em conjunto com outras variáveis, e não de

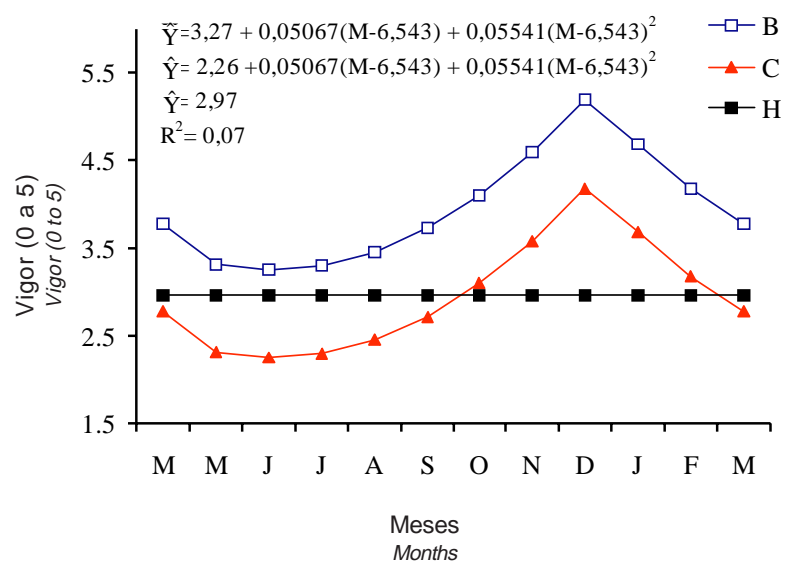

Figura 3 - Comportamento do vigor espermático de carneiros das raças $\mathrm{Be}$, Co e $\mathrm{Hd}$, em relação aos meses do ano.

Figure 3 - The behaviour of sperm vigor of rams of $\mathrm{Be}, \mathrm{Co}$ and $\mathrm{Hd}$ breeds, on months of the year.

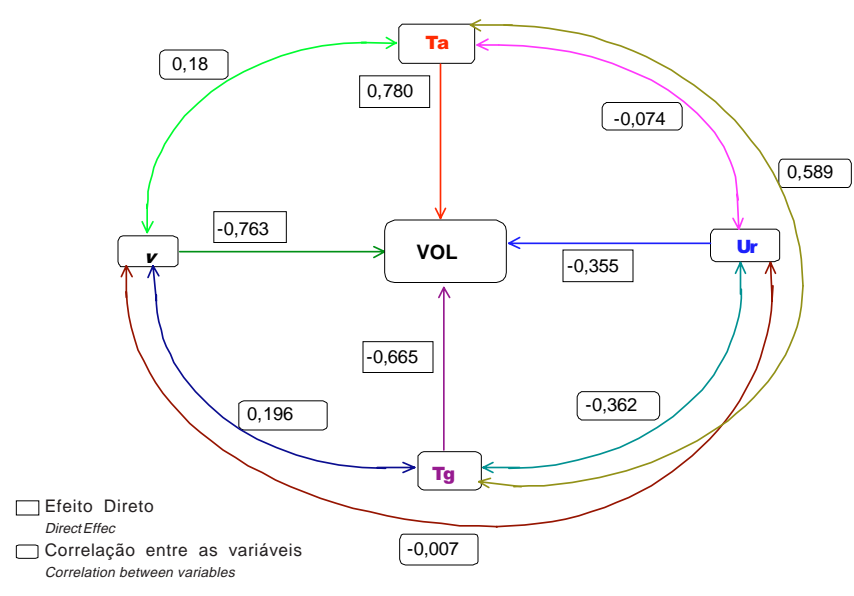

Figura 4 - Diagrama das estimativas de correlação entre as variáveis climáticas e de seus efeitos diretos sobre o volume do sêmen de carneiros das raças $\mathrm{Be}$, Co e Hd.

Figure 4 - Diagram of the correlation between the climatic parameters and their direct effects estimates on the semen volume of rams of $\mathrm{Be}$, Co and $\mathrm{Hd}$ breeds.

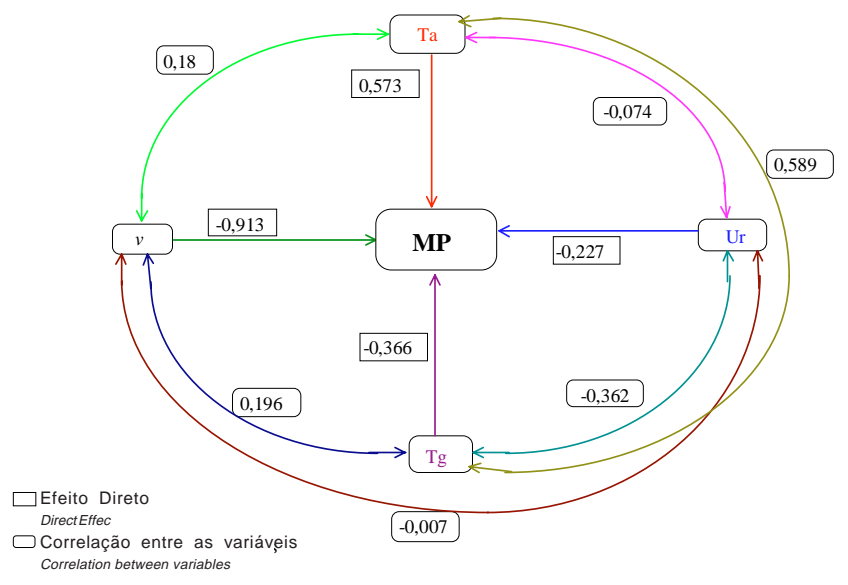

Figura 5 - Diagrama das estimativas de correlação entre as variáveis climáticas e de seus efeitos diretos sobre a MP espermática de carneiros das raças $\mathrm{Be}$, Co e Hd.

Figure 5 - Diagram of the correlation between the climatic parameters and their direct effects estimates on the sperm PM of rams of $\mathrm{Be}, \mathrm{Co}$ and $\mathrm{Hd}$ breeds.

forma isolada. A Ur apresentou efeito direto reduzido e baixa correlação, indicando ser de pouca utilidade para a estimativa desta característica.

A Figura 5 mostra os efeitos das variáveis climáticas sobre a MP. A $v$ apresentou efeito direto de 0,913 , enquanto seus efeitos indiretos via $\mathrm{Ta}, \mathrm{Tg}$ e $\mathrm{Ur}$ foram de 0,103 ; $-0,071$; e 0,001 , respectivamente. A Ta apresentou efeito direto de 0,573 , enquanto seus 
efeitos indiretos via Tg, ve Ur foram de -0,215;-0,164; e 0,016 , respectivamente. A $\mathrm{Tg}$ apresentou efeito direto de $-0,366$ e seus efeitos indiretos via Ta, $v$ e Ur foram de 0,$337 ;-0,178$; e 0,082 , respectivamente. A Ur apresentou efeito direto de $-0,227$ e seus efeitos indiretos via $\mathrm{Tg}$, Ta e $v$ foram de 0,$132 ;-0,042$; e 0,006 , respectivamente.

A $v$ apresentou alto efeito direto e correlação com a MP, determinando a variação. A Ta e a Tg apresentaram, respectivamente, alto e moderado efeito direto e baixa correlação, sendo mais benéficas para as estimativas quando em conjunto com outras variáveis, e não de forma isolada. A Ur apresentou efeito direto reduzido e baixa correlação, indicando ser de pouca utilidade para a estimativa desta característica.

A Figura 6 mostra os efeitos das variáveis climáticas sobre o vigor.

A $v$ apresentou efeito direto de $-0,861$ e seus efeitos indiretos via Ta, Tg e Ur foram de 0,127; $-0,085$; e 0,001 , respectivamente. A Ta apresentou efeito direto de 0,711 e seus efeitos indiretos via $\mathrm{Tg}$, $v$ e Ur foram de $-0,257$; $-0,154$; e 0,016 , respectivamente. A $\mathrm{Tg}$ apresentou efeito direto de -0,437, enquanto seus efeitos indiretos via Ta, $v$ e Ur foram de 0,$418 ;-0,168$; e 0,08 , respectivamente. A Ur apresentou efeito direto de $-0,223$ e seus efeitos indiretos via $\mathrm{Tg}$, Ta e $v$ foram de 0,158; -0,052; e 0,005 , respectivamente. A $v$ apresentou alto efeito direto e correlação com o vigor, determinando a variação. A Ta e Tg apresentaram, respectivamente,

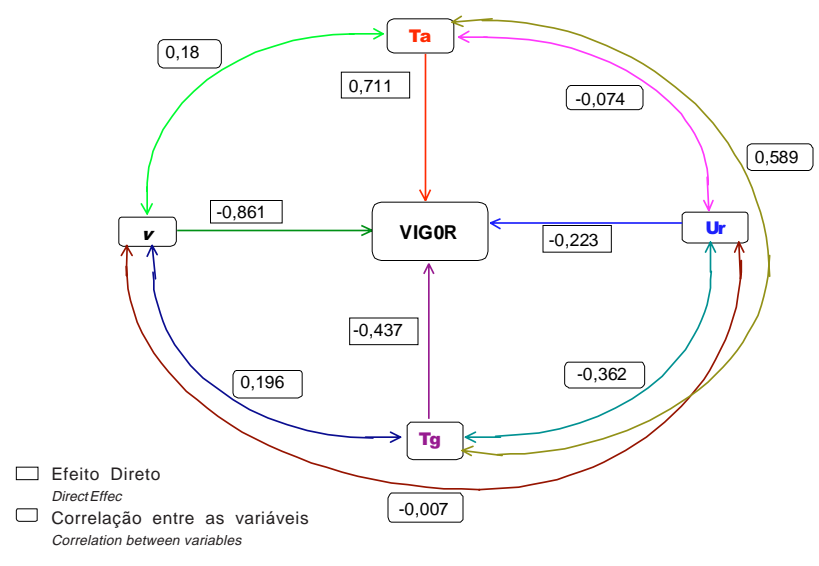

Figura 6 - Diagrama das estimativas de correlação entre as variáveis climáticas e de seus efeitos diretos sobre o vigor espermático de carneiros das raças $\mathrm{Be}$, Co e $\mathrm{Hd}$.

Figure 6 - Diagram of the correlation between the climatic parameters and their direct effects estimates on the sperm vigor of rams of Be, Co and $\mathrm{Hd}$ breeds. alto e moderado efeito direto e moderada e baixa correlação com o vigor, sendo mais benéficas para as estimativas quando em conjunto com outras variáveis, e não de forma isolada. A Ur apresentou efeito direto reduzido e baixa correlação, indicando ser de pouca utilidade para a estimativa desta característica.

\section{Conclusões}

As variações encontradas nas características do sêmen das raças estudadas, durante as estações do ano, ocorreram em função de sua estacionalidade reprodutiva.

As raças Bergamácia e Hampshire Down apresentaram melhores respostas para as características do sêmen que a raça Corriedale, demonstrando sua melhor resistência ao ambiente.

Das variáveis climáticas estudadas, a velocidade do vento $(v)$ foi a variável que apresentou maior influência sobre as características do sêmen, seguida da temperatura do ar e temperatura do globo e, por último, a umidade relativa do ar que apresentou menor influência. Assim, especial atenção deve ser dada à variável velocidade do vento no sentido de permitir que os animais utilizem este mecanismo termorregulador com melhor eficiência, resultando em melhor qualidade do sêmen na fase reprodutiva.

\section{Referências Bibliográficas}

BOLAND, M.P., AL-KAMALI, A.A., CROSBY, T.F. et al. 1985. The influence of breed, season and photoperiod on semen characteristics, testicular size, libido and plasma hormone concentrations in rams. Anim. Reprod. Sci., 9:241-252.

BROOKS, J.R., ROSS, C.V. 1962. Effect of ambient temperature and thyroxine therapy on semen quality of rams. J. Anim. Sci., 21:700-705.

CHAHAL, A.S., RATTAN, P.J.S., PARSHAD, O. 1979. Some physico-chemical studies on semen and their interrelationship during different seasons in Corriedale rams. Ind. J. Anim. Sci., 49:433-437.

CHEMINAU, P. 1994. Environment and animal reproduction. World Anim. Rev., 77:2-14.

CRUZ, C.D., REGAZZI, A.J. 1994. Modelos biométricos aplicados ao melhoramento genético. Viçosa: Universidade Federal de Viçosa, Imprensa Universitária. 390p.

CURTIS, S.E. 1983. Environmental management in animal agriculture. ed. Iowa State: University Press. 409p.

DERIVAUX, J. 1976. Reproduccion de Los Animales Domesticos. 2. ed. Espanha: Zaragoza. 486p.

DRAPER, N.R., SMITH, H. 1981. Applied regression analysis, 2. ed. New York: John Wiley \& Sons. 709p.

DUFOUR, J.J., FAHMY, M.H., MINVIELLE, F. 1984. Seasonal changes in breeding activity, testicular size, testosterone concentration and seminal characteristics in rams with long 
Rev. bras. zootec.

or short breeding season. J. Anim. Sci., 58:416-422.

DUTT, R.H., BUSH, L.F. 1955. The effect of low environmental temperature on initiation of the breeding season and fertility in sheep. J. Anim. Sci., 14:885-897.

DUTT, R.H., SIMPSON, E.C. 1957. Environmental temperature and fertility of Southdown rams early in the breeding season. J. Anim. Sci., 16:136-143.

FOOTE, R.H. 1982. Inseminação artificial. In: HAFEZ, E.S.E. Reprodução animal. 4. ed. São Paulo. 720p.

GRAYBILL, F.A. 1976. Theory and application of the linear model. Massachusetts: Duxbury Press. 704p.

HULET, C.V., SHELTON, M. 1982. Ovinos e caprinos. In: HAFEZ, E.S.E. Reprodução animal. 4. ed. São Paulo. 720p.

MICKELSEN, W.D., PAISLEY, L.G., DAHMEN, J.J. 1981. Scrotal circumference and sperm motility and morphology in rams. Theriogenology, 16:45-51.

MIES FILHO, A. 1987. (Ed.) Reprodução dos animais domésticos e inseminação artificial. 6. ed., v.I e II, Porto Alegre. RS: Editora Sulina S/A. 645p.

MITTAL, J.P., GHOSH, P.K. 1979. Comparative semen characteristics of Corriedale, Marwari, and Jaisalmeri rams maintained under hot arid conditions. J. Agric. Sci., 92:1-4.

MOULE, G.R. 1965. Field investigation with sheep - A manual of techniques. The commonwealth Scientific and Industrial Research Organization. Austrália. 480p.
PINEDA, M.H. 1989. Male reproduction. In: McDONALD, L.E., PINEDA,M.H. (Eds.) Veterinary endocrinology and reproduction. 4. ed. Philadelphia, London: Lea \& Fasiger. 571p.

RAO, B.R., PANDEY, J.N., JAIRAM, B.T. 1983. Seasonal variation of certain biochemical constituents in the semen of Nali and Corriedale rams and their relationship with physical attributes under semi-arid tropical regions. Ind. Vet. J., 60:199-204.

SILVA, M.A. 1980. Melhoramento animal (conceitos básicos da análise de dados). Viçosa. Imprensa Universitária. 81p.

SILVA, R.G. Equações para estimar a carga térmica radiante através do globo negro. In: WORKSHOP BRASILEIRO DE BIOCLIMATOLOGIA ANIMAL, 2, 1989, Jaboticabal. Anais... Jaboticabal: UNESP, 1989, p.1-9.

SILVA, R.G., BRASIL, D.F. 1986. Calibração de catatermômetro para avaliação de pequenos deslocamentos de ar. Ciências Zootécnicas, 1:1-3.

Recebido em: 10/03/98

Aceito em: 08/02/99 